\title{
Stokes imaging polarimetry using image restoration at the Swedish 1-m solar telescope
}

\author{
M. J. van Noort ${ }^{1}$ and L. H. M. Rouppe van der Voort ${ }^{2, \star}$ \\ ${ }^{1}$ Institute for Solar Physics of the Royal Swedish Academy of Sciences, AlbaNova University Center, 10691 Stockholm, Sweden \\ e-mail: noort@astro.su.se \\ 2 Institute of Theoretical Astrophysics, University of Oslo, PO Box 1029 Blindern, 0315 Oslo, Norway \\ e-mail: rouppe@astro.uio.no
}

Received 13 April 2008 / Accepted 2 June 2008

\begin{abstract}
Aims. We aim to achieve both high spatial resolution and high polarimetric sensitivity, using an earth-based $1 \mathrm{~m}$-class solar telescope, for the study of magnetic fine structure on the surface of the Sun.

Methods. We use a setup with 3 high-speed, low-noise cameras to construct datasets with interleaved polarimetric states, particularly suitable for Multi-Object Multi-Frame Blind Deconvolution image restorations. We discuss the polarimetric calibration routine and various potential sources of error in the results.

Results. We obtained near diffraction limited images, which have a noise level of $\approx 10^{-3} I_{\text {cont }}$. We confirm that dark cores have a weaker magnetic field and a lower inclination angle with respect to the solar surface than the edges of the penumbral filament. We demonstrate that the magnetic field strength in faculae-striations is significantly lower than in other nearby parts of the faculae.
\end{abstract}

Key words. methods: observational - techniques: image processing - techniques: polarimetric - Sun: magnetic fields - sunspots Sun: faculae, plages

\section{Introduction}

Since the discovery of magnetic features on the Sun (Hale 1908), polarimetry has become an increasingly important diagnostic tool for solar observations. The primary goals in resolution, required signal-to-noise ratio $(\mathrm{S} / \mathrm{N})$ and spectral resolution have continually shifted with the objects of interest, from strong, large-scale fields to smaller and often weaker structures.

Considerable efforts have been made toward meeting these requirements. Examples of modern instruments at some of the major solar observing facilities are the DiffractionLimited Spectro-Polarimeter (DLSP, Sankarasubramanian et al. 2004), the Tenerife Infrared Polarimeter (TIP, Mártinez Pillet et al. 1999; Collados et al. 2007), the Polarimeteric Littrow Spectrograph (POLIS, Beck et al. 2005b), and the multi-line spectropolarimetric (MTR) observing mode at Themis (Paletou \& Molodij 2001). Typical spatial resolution achieved with these instruments is of order $0{ }^{\prime} 5-1$.".

Major progress has been made by the spectro-polarimeter (SP, Lites et al. 2001) on the Solar Optical Telescope (SOT, Suematsu et al. 2008) onboard the Hinode spacecraft. The combination of $\sim 0$ !' 3 spatial resolution and $10^{-3}-10^{-4}$ relative sensitivity is defining new standards in solar polarimetry. The excellent performance of the image stabilizer of SOT enables the SP to exploit fully one of the major advantages of space observations: the absence of seeing.

Obtaining ground based solar image data suitable for producing sensitive full Stokes maps presents a significant challenge. The problem poses two important requirements on the data, which are difficult to accommodate at the same time. To

\footnotetext{
* Also at the Center of Mathematics for Applications, University of Oslo, PO Box 1053 Blindern, 0316 Oslo, Norway.
}

maximize resolution, as short as possible exposure times are desirable to minimize smearing by seeing-induced image motions. To maximize sensitivity, as long as possible exposure times are needed to minimize the photon noise in the images. Obviously these two requirements are mutually exclusive so that a suitable compromise must be made.

Several approaches currently exist toward addressing one or both of these problems, two of which have been used extensively. The first approach has been very successful at eliminating artificial signals from seeing-induced image motions, by means of high-frequency modulation of the polarimetric state, and integration in all polarimetric states for a long time. As the seeing-induced image aberrations are identical for all intents and purposes, artifacts resulting from image-motions are essentially eliminated. Using this method, a relative sensitivity of $10^{-5}$ was achieved by the Zürich Imaging Stokes Polarimeter II (ZIMPOL, Gandorfer \& Povel 1997). Such extreme levels of sensitivity can currently only be achieved at the cost of losing a great deal of spatial resolution.

Another approach is the removal of seeing-induced image aberrations by numerical means. This approach attempts to address both problems at once, but requires frequent CCD readouts to construct an image stack for analysis. Consequently, it is of particular importance to ensure that the photon noise exceeds the readout noise of the CCD, to achieve a good $\mathrm{S} / \mathrm{N}$ in the end.

Bello González \& Kneer (2008) achieved a polarimetric sensitivity of $10^{-3}$ at a spatial resolution of $\approx 0^{\prime} 3$ using a combination of adaptive optics and speckle reconstruction. Their observing method elaborates on the pioneering work of Keller \& von der Lühe (1992) at the Swedish Vacuum Solar Telescope, where they introduced the use of a narrow-band channel in combination with a wide-band channel. The high light levels in 
the wide-band channel enabled them to successfully perform speckle restorations, after which the seeing information acquired from the wideband restorations was used to restore the narrowband images.

The Multi-Object Multi-Frame Blind Deconvolution (MOMFBD) post-processing method used by van Noort et al. (2005), like the speckle method used by Keller \& von der Lühe (1992), combines the signal accumulated in a large number of short-exposure images using an image restoration process that separates the image information from the seeing-induced image blurring and distortions.

Although more expensive numerically than speckle reconstruction, some advantages of the MOMFBD method are that: some fixed (instrumental) aberrations can be corrected using a phase-diversity channel; the process uses all image channels for wavefront sensing, resulting in a more accurate separation of image information and seeing distortions; and the requirements for the image data are less stringent in terms of the statistical independence of individual exposures and the seeing statistics in general, so that it can be easily applied to very high cadence data, recorded using an adaptive optics system.

\section{Full Stokes measurements at the SST}

To acquire accurate polarimetric data, a reliable polarimeter is needed. Although the entire telescope is part of the polarimeter, it is more practical to separate the vacuum part of the telescope from the remainder of the optical setup and model it separately.

The reason for this is that the design of the Swedish $1 \mathrm{~m}$ Solar Telescope (SST, Scharmer et al. 2003a) includes vacuum windows and large angle of incidence mirrors, so that the instrumental polarization is not only considerable, at $\approx 10 \%$ in both Stokes $Q$ and $U$ and around $2 \%$ in Stokes $V$, it also depends on the mirror angles and thus on the observing position in the sky and is therefore time dependent.

Since the determination of the telescope matrix for all possible positions in the sky is not practically achievable (although not impossible), it makes sense to model the telescope and calibrate the model parameters instead. For this, however, an accurate polarimeter is required.

\subsection{Polarimeter}

The polarimeter at the SST is similar to that used by Horn \& Hofmann (1999) and consists of two Liquid Crystal Variable Retarders (LCVR), positioned in the beam, the first aligned with a vertical analyzer polarization filter, which is part of the SOUP filter and the second at a $45^{\circ}$ angle with respect to the first. By varying the retardance of each LCVR and filtering the resulting "modulated" light through an analyzer Linear Polarizer (LP), the polarimeter can be configured to transmit only certain (linearly independent) combinations of the input Stokes parameters. The conversion coefficients from Stokes parameters to transmitted intensity are the elements of the modulation matrix $M$, the inverse of which, the demodulation matrix, must be known to retrieve the input Stokes parameters.

The choice of LCVRs over the more commonly used FerroElectric LCs, is motivated by the greater flexibility in choosing the modulation scheme and effortless adaptation to changes in the observing wavelength. The price to pay for this flexibility is a "slow" response to changes in modulation value $(\sim 10 \mathrm{~ms}$ or more) when an increase in retardance is required, which presents a problem when combined with high-speed cameras, as well as stringent stability requirements on the driving voltage, especially at the high end of the retardance range of the LCVRs, where the sensitivity to voltage changes is largest.

The speed restrictions of the LCVRs can be effectively dealt with using an "overdrive" approach: when an increase in retardance is needed, the driving voltage amplitude is set to 0 , to maximize the rate of change. When the desired retardance value is reached (or passed), the voltage appropriate to the target retardance value is applied, upon which the LCVR has only a small, negative, retardance change to cover, which can be accomplished more than an order of magnitude faster than for an equal positive change. In combination with optimizing the state order of the modulation scheme, the tuning time of the LCVRs can be kept below $10 \mathrm{~ms}$ at all times, a requirement imposed by the readout time of the cameras used.

Since the retardance value of Liquid Crystals is also temperature sensitive, the ability to continuously vary the retardance value is an advantage: it can be used to correct the retardance based on the measured temperature, so that complications related to thermal stabilization can be avoided.

The current implementation, however, still makes use of a simple thermal stabilization circuit, which, although it improves considerably upon the unstabilized situation, is not able to cope with sudden changes in the environmental conditions, such as those arising from changes in ventilation. Therefore, although the polarimeter is currently operational, its properties are slowly varying over time and need to be recalibrated as frequently as possible. Once the stability issues are properly addressed, this should not be needed, as long as the optical setup remains the same.

For most of the data presented here, the polarimeter could be calibrated within a few hours of taking the data, but uncorrected variations in the measured Stokes parameters at the level of approximately one percent usually remain.

\subsection{Polarimeter calibration}

To calibrate the polarimeter, a sufficiently large number of linearly independent Stokes vectors needs to be generated. This is achieved by using a combination of a Linear Polarizer (LP) and a Quarter Wave Plate (QWP): a $90^{\circ}$ linear retarder. The LP is positioned at several fixed angles, after which the retarder is rotated through $360+^{\circ}$, such that some of the linearly polarized light is converted to other Stokes parameters according to

$$
\begin{gathered}
\quad S(\varphi, \alpha)= \\
\frac{1}{2}\left(\begin{array}{cccc}
1 & 0 & 0 & 0 \\
0 & \cos ^{2} 2 \alpha+\sin ^{2} 2 \alpha \cos \delta & \cos 2 \alpha \sin 2 \alpha(1-\cos \delta) & -\sin 2 \alpha \sin \delta \\
0 & \cos 2 \alpha \sin 2 \alpha(1-\cos \delta) & \sin ^{2} 2 \alpha+\cos ^{2} 2 \alpha \cos \delta & \cos 2 \alpha \sin \delta \\
0 & \sin 2 \alpha \sin \delta & -\cos 2 \alpha \sin \delta & \cos \delta
\end{array}\right) \\
\\
\quad\left(\begin{array}{cccc}
1 & \cos (2 \varphi) & \sin (2 \varphi) & 0 \\
\cos (2 \varphi) & \cos ^{2}(2 \varphi) & \cos (2 \varphi) \sin (2 \varphi) & 0 \\
\sin (2 \varphi) & \cos (2 \varphi) \sin (2 \varphi) & \sin ^{2}(2 \varphi) & 0 \\
0 & 0 & 0 & 0
\end{array}\right) S_{\text {in }}
\end{gathered}
$$

where $S_{\text {in }}$ is the input Stokes vector; $S(\varphi, \alpha)$ is the generated Stokes vector; $\varphi$ and $\alpha$ are the offset angle of the LP and the retarder, respectively, with respect to the positive Stokes $Q$ axis; and $\delta$ is the retardance value of the QWP.

For calibration purposes, the LP was positioned at $0^{\circ}, 45^{\circ}$, $90^{\circ}$, and $135^{\circ}$, coinciding with the $Q, U,-Q$, and $-U$ axes respectively. This ensures that all Stokes components are present at their maximum amplitudes and with opposite sign, to reduce sensitivity to offsets and other sources of contamination in the calibration data. Although a condition number analysis of the problem by Selbing (2005) suggests that angle steps of $30^{\circ}$ over 


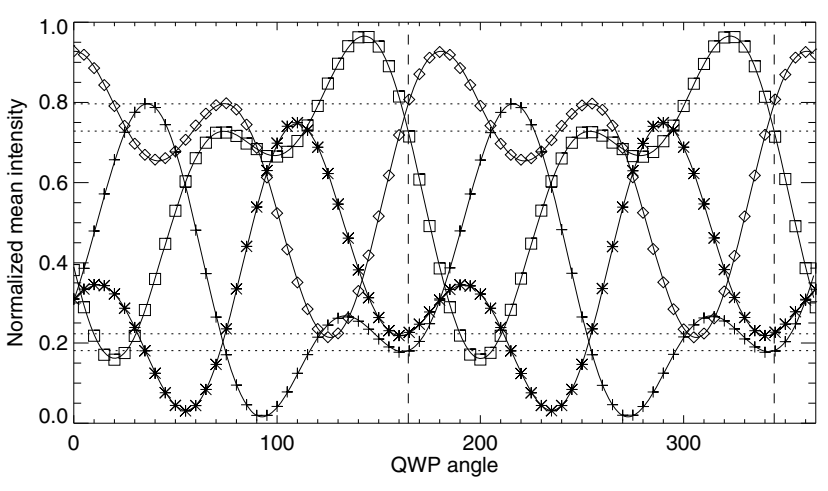

Fig. 1. Polarimetry Calibration for 13-Sep.-2007. The lines are theoretical curves fitted to the data recorded for the polarimetric states. The horizontal dotted lines are the LP-only observations, the vertical dashed lines are the fitted angle offset (for $0^{\circ}$ and $180^{\circ}$ ) of the QWP. The accuracy with which the intersection points of the LP-only lines and their corresponding curves line up vertically and how well this matches the fitted offset angle is an indication of the overall accuracy of the fit.

a range of only $180^{\circ}$ should be sufficiently accurate to determine fully the problem, the angles for the retarder were taken to be every $5^{\circ}$ from $0^{\circ}$ to $365^{\circ}$, a total of 74 angles, to average out any error in the QWP angles and photon noise in the data that may exist.

Strictly speaking, the modulation matrix elements can now be fitted by a simple matrix inversion. However, this would assume that the retardance value and offset angle of the QWP are known perfectly, as well as that the intensity was constant during the recording of the calibration data. An iterative approach is the most obvious way forward because the expressions containing the retarder parameters are non-linear and, to compensate for the varying intensity, the observations must be normalized to $I$, for which the inverse of the modulation matrix itself needs to be known.

Using a downhill gradient-search algorithm for all parameters is the simplest way to find the solution, but it is rather slow and can benefit greatly from step direction optimization methods such as conjugate-gradient optimization. Unfortunately, it is easy to end up in a local minimum close to the global minimum. An improvement can be made by using as much of the explicit solution as possible $(M)$ and using a downhill search algorithm for the remaining parameters ( $\alpha$ and $\left.\delta_{\mathrm{QWP}}\right)$. Although this method converges far more rapidly and is less likely to find a local minimum, it is more likely to end up with a completely wrong solution if the start solution is far away from the real solution. However, both methods are based on minimizing the same error function and converge toward the same solution, if sufficiently restrictive convergence criteria are used.

In addition to the calibration data, observations were made without the QWP. This provides an independent way of checking the quality of the fit, since the fitted data curves should assume the value of the LP-only observations at the retarder angle equal to that of the LP.

Figure 1 shows an example of a calibration data set with fitted model curves, for the mean over the field of view. Typically, a total of 8 of these curves are used to fit the modulation matrix elements. The QWP angle for which the curves assume the value of the LP-only observations is the same for all curves to within $0.1^{\circ}$, but differs from the calibrated QWP offset angle by $0.45^{\circ}$, possibly due to inaccuracies introduced by the manual positioning of the LP, the positions of which were not included in the fit.

From the modulation matrix produced by the calibration, efficiencies of $0.56,0.53$, and 0.51 for Stokes $Q, U$, and $V$ respectively can be derived. The total efficiency of 0.94 indicates that the modulation scheme is close to the theoretical optimum.

\subsection{Telescope model}

Since the telescope uses 2 mirrors at a $45^{\circ}$ angle of incidence and a vacuum-window, it is strongly polarizing and the polarization depends on the pointing direction. To predict the Mueller matrix of this system for arbitrary azimuth and elevation angles, it needs to be modeled and then calibrated. The model is based on the method used by Skumanich et al. (1997), a method that was applied successfully to model the DST, the SVST (LPSP, Sanchez Almeida et al. 1997; Mártinez Pillet et al. 1999), and the German VTT (Beck et al. 2005a). Selbing (2005) adapted the method to describe the SST optics.

The Mueller matrix of the telescope is the product of the series of Mueller matrices for each of the optical components the light passes through before exiting the bottom of the telescope

$M_{\mathrm{tel}}=M_{\text {Schup }} \cdot M_{\text {field }} \cdot R_{\mathrm{az}} \cdot M_{\mathrm{az}} \cdot R_{\mathrm{el}} \cdot M_{\mathrm{el}} \cdot L$,

where the main objective lens matrix is $L$, the folding mirrors are $M_{\mathrm{el}}$ and $M_{\mathrm{az}}$, the field mirror is $M_{\text {field }}$ at the bottom of the telescope, and the Schupmann corrector mirror is $M_{\text {Schup }}$, with appropriate rotations $R$ to the natural frame of the next component.

The corresponding matrix is obviously position dependent, but this is through the rotation matrices $R$ only, so that this dependence is known once the matrices for all other components are known. The mirror matrices can be described using a general expression with only 2 unknown parameters, so that only $L$ and a few parameters need to be calibrated. This can only be done by fitting the predicted telescope response to known input Stokes data.

Unfortunately, since the entrance window has a $1 \mathrm{~m}$ diameter, it is not possible to use the method of generating Stokes vectors used when calibrating the polarimeter, since a $1 \mathrm{~m}$ retarder of sufficient quality is hard to obtain. Only a fit to linearly polarized light is possible.

To provide a source of linearly polarized light, a large sheet of polarizing material was mounted on a rotating wheel in front of the telescope main lens. This wheel was then rotated slowly and continuously while pointing toward the Sun, thus presenting the telescope with all Stokes parameters apart from Stokes $V$. Polarimetric data were acquired continuously throughout the day, to provide information on the azimuth and elevation dependence of the telescope matrix.

Although of fundamental importance to the accuracy of the Stokes data presented here, further details of the telescope calibration procedure are beyond the scope of this paper and are outlined in detail by Selbing (2005). We limit ourselves to stating that the estimated error in the predicted output of the telescope was found to be below $0.5 \%$ in Stokes $Q, U$, and $V$, based on the only calibration performed so far, in March 2004. However, since there is no information available about the effects of aging and maintenance on the model parameters, this estimate may no longer be accurate.

Figure 2 shows the instrumental polarization estimated from a full day of full Stokes data recorded on 12 Sep. 2006, more than a year after the telescope model was calibrated. Since the 


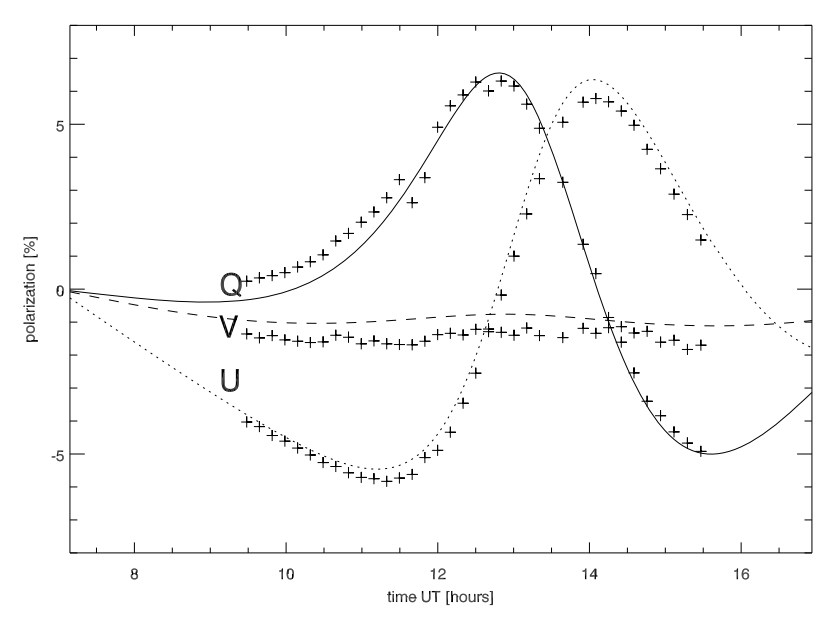

Fig. 2. Instrumental polarization for 12-Sep.-2006 as a function of time. The plotted curves are the model predicted telescope polarization for $Q$ (solid), $U$ (dotted) and $V$ (dashed), the crosses are the actual values measured below the exit window of the telescope.

FOV is dominated by a large sunspot close to the center of the solar disc, the derived offset for each Stokes parameter may contain a non-instrumental component of solar origin. Although the model predictions show a small systematic difference from the data, in particular the extreme values of $Q$ and $U$ appear to be assumed at a somewhat different time of day from the model, the agreement with the telescope model appears to be closer than approximately $1 \%$ of $I$, which is not significantly poorer than found 18 months earlier.

\section{Observations and data reduction}

From several campaigns during the 2006 observing season, we selected 4 data sets of sunspots that were taken under excellent seeing conditions. Detailed information about the different targets is presented in Table 1. Figure 3 shows the four Stokes maps for FeI $630.25 \mathrm{~nm}-5 \mathrm{pm}$ for one of the selected sunspots.

The quality of the data benefitted from the SST adaptive optics system (AO, Scharmer et al. 2003b) and post-processing using the Multi-Object Multi-Frame Blind Deconvolution (MOMFBD, van Noort et al. 2005) image restoration method. The use of MOMFBD is complementary to the use of AO in the sense that it provides the possibility to simultaneously achieve high image quality close to and at large angular distances away from the $\mathrm{AO}$ wavefront sensor.

Narrow band images in the wings of the FeI $630.25 \mathrm{~nm}$ line were obtained with the Solar Optical Universal Polarimeter (SOUP, Title \& Rosenberg 1981), which has a FWHM of $7.2 \mathrm{pm}$. Three science cameras were employed with $1024 \times 1024$ pixel CCD chips (pixel scale 0.'063, the diffraction limit $\lambda / \mathrm{D}$ of the SST at $630 \mathrm{~nm}$ is $0{ }^{\prime} 13$ ). One of these cameras was positioned behind the SOUP filter and the other two were set-up as a phasediversity pair that received $7.5 \%$ of the light on a branch split off the main beam before the SOUP but after the SOUP prefilter $(0.8 \mathrm{~nm}$ passband centered on $630.25 \mathrm{~nm})$. The exposures of all three cameras were synchronized by means of an optical chopper. The optical setup was similar to the one described in De Pontieu et al. (2007) with an additional pair of LCVRs positioned between the collimator and re-imaging lens, and is illustrated in Fig. 4.
Since the polarimetric signals of interest are usually weak, it is of crucial importance to obtain the largest $S / N$ ratio possible. Although the Sun is an excellent source of photons, when reducing the spectral range to only a few tens of pm, acquiring a detectable quantity of photo electrons in the few ms permitted by the seeing becomes a challenge. To take full advantage of the available light, the cameras used at the SST are Sarnoff 1M100 cameras, the CCD of which has a quantum efficiency of 0.7 at $630 \mathrm{~nm}$ and consists of 16 separate segments that can be read out in parallel. By using a slow pixel clock, the result is a camera with exceptionally low readout noise and a readout time of only $10 \mathrm{~ms}$. Even with this short readout time, the most effective exposure time is a compromise between as short as possible, to "freeze" the seeing, and at least longer than the readout time, to achieve a reasonable efficiency. In practice, the rotation rate was set to be approximately $36 \mathrm{~Hz}$, giving an exposure time of $\sim 17 \mathrm{~ms}$ and a duty-cycle of approximately $60 \%$. Apart from the desire to have a large duty-cycle, a shorter exposure at the current light levels (using the SOUP filter which has a transmission of only $\sim 15 \%$ ) would result in a photon noise that is at or below the readout noise of the camera, a situation that the MOMFBD reduction is currently unable to handle.

The LCVRs of the polarimeter cycled through a sequence of 4 states, changing state for each exposure during the read-out time of the cameras. The SOUP filter ran a program switching between the red and blue wing of the FeI $630.25 \mathrm{~nm}$ line, at $\pm 5 \mathrm{pm}$ offset from the line core. A change of line position was made every $\sim 14 \mathrm{~s}$, a period covering typically 125 LCVR cycles. With a line position changing time of about $7 \mathrm{~s}$, the acquisition of a full data set, containing two line positions and all LCVR cycles, was completed in about $35 \mathrm{~s}$. The exact number of images (and effective exposure time) for each data set is specified in Table 1.

Figure 5 illustrates the acquisition scheme of the data. Since the cameras are synchronized, 3 exposures share the same wavefront realization $t$ ( $x$-axis): one SOUP image with a specific line position and LCVR state $o$ ( $z$-axis), and two wide-band images with known diversity $k$ ( $y$-axis), one in focus and one slightly out of focus ( $\sim 12 \mathrm{~mm}$ on a $45 \mathrm{~m}$ focal length).

The figure is similar to Fig. 7 in van Noort et al. (2005) with the important difference that the LCVR states are now changed for each exposure instead of following a burst of several exposures. This produces a collection of polarimetric states that is more homogeneous in terms of seeing aberrations, so that the level of seeing cross-talk is already reduced significantly prior to MOMFBD processing. The interleaved character of the data set is akin to the high speed modulation used by the Zürich Imaging Polarimeter (ZIMPOL, Gandorfer \& Povel 1997), which employs modulation in the $\mathrm{kHz}$ range and is highly beneficial for the construction of high-sensitivity Stokes maps.

The images from the wide-band cameras vary only because of seeing variations, while images from the SOUP camera vary constantly due to seeing variations, the modulation of the LCVRs, and the changing line position. Since the wavelength of the wide-band images is almost identical to that of the narrowband images, the wide-band images can be used as an "anchor" channel, to which all restored SOUP objects are aligned in the MOMFBD restoration procedure as discussed at length in van Noort et al. (2005).

To calibrate the inter-camera alignment, images of an array of $30 \mu \mathrm{m}$ pinholes were recorded. By fitting these pinhole images to each other and assuming that they are the same, the relative offset of each camera pixel with respect to a properly chosen reference camera can be calculated using the MOMFBD 

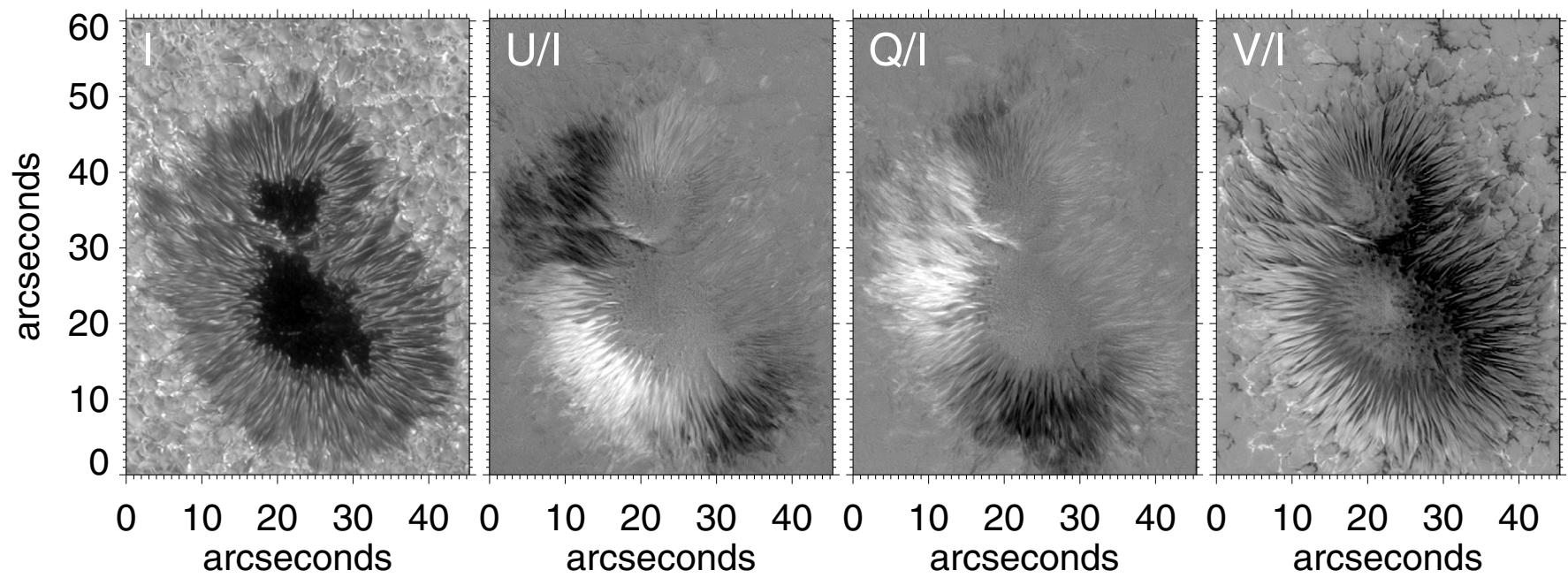

Fig. 3. Sunspot AR10908 observed on 12 Sep. 2006 in FeI $630.25 \mathrm{~nm}-5 \mathrm{pm}$. The color scaling saturates at $\pm 15 \%$ for U/I and Q/I, and at $\pm 25 \%$ for $\mathrm{V} / \mathrm{I}$.

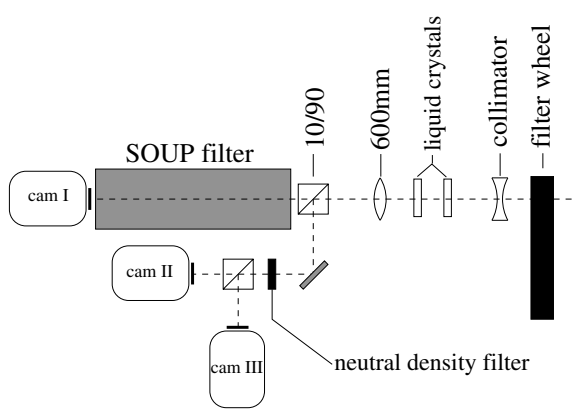

Fig. 4. Schematic drawing of the optical set-up.

Table 1. Summary of observed targets. $\mu=\cos (\theta)$ with $\theta$ the observing angle. e time is the effective exposure time per line position, i.e., the sum of all exposure times.

\begin{tabular}{ccccc}
\hline \hline date & NOAA number & position [ $\left.{ }^{\circ}\right]$ & $\mu$ & e time \\
\hline 07-Jan-2006 & AR10845 & N18 E64 & 0.39 & 7.8 \\
10-Jun-2006 & AR10893 & N01 E17 & 0.95 & 7.8 \\
12-Sep-2006 & AR10908 & S09 W12 & 0.94 & 7.5 \\
15-Sep-2006 & AR10908 & S13 W51 & 0.58 & 7.7 \\
\hline
\end{tabular}

code in calibration mode. These image offset calibrations result in the alignment of restored objects with a typical accuracy of $\approx 0.05$ pixels. This means that the restored SOUP objects have near-perfect alignment - even though the individual exposures are not recorded simultaneously - and no further alignment by means of cross-correlation is needed to construct the Stokes maps. The level of false signals induced by seeing is therefore generally very low (see also Sect. 4.1).

Unless otherwise stated, all images were reduced using $128 \times$ $128\left(5 \times 5^{\prime \prime}\right)$ and seamless mosaicking of the tapered subimages. The reductions were done on a 90 CPU 3.2 GHz Intel Xeon cluster at the University of Oslo using 34 Karhunen-Loéve wavefront modes and converged until the relative change in the wavefront coefficients was $<10^{-4}$.

\section{Quality assessment}

\subsection{Noise}

One of the main motivations for conducting observations in the way that we describe in this paper is the detection of weak

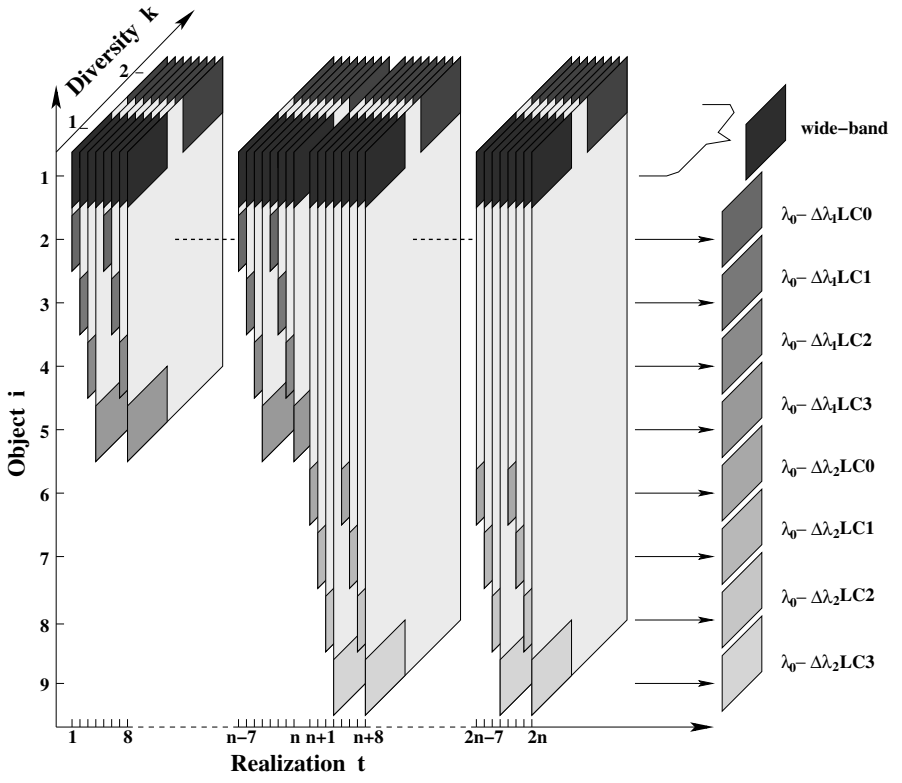

Fig. 5. Sketch of the acquisition scheme of a full Stokes MOMFBD data set for 2 line positions. The 2 line positions, $\lambda_{0}-\Delta \lambda_{1}$ and $\lambda_{0}-\Delta \lambda_{2}$, were recorded sequentially. For each line position, the LCVRs cycled through 4 states LC0-LC3, changing state for each exposure $t$. For each narrowband exposure, a pair of accompanying wide-band phase-diversity images were recorded. The wide-band channel served as an anchor channel for the narrow-band images. For the data sets discussed in this paper, the number of exposures for each line position was 500 , i.e., $n=500$.

polarization signals. It is therefore important to quantify accurately the noise level in the data. The main source of noise is photon noise. Read-out noise is a minor source of noise: for the Sarnoff cameras, the read-out noise is specified by the manufacturer to be on the order of 25 electrons, which amounts to 1-2 counts. Seeing cross-talk is another potential source of noise. As discussed in Sect. 3, the use of a wide-band anchor channel in MOMFBD restorations reduces seeing cross-talk significantly in derived quantities such as magnetograms. The use of interleaved LCVR state exposures, in addition, reduces yet more the effect of seeing cross-talk.

Even though, images free from polarization signal are needed to investigate the noise, there is no location on the 


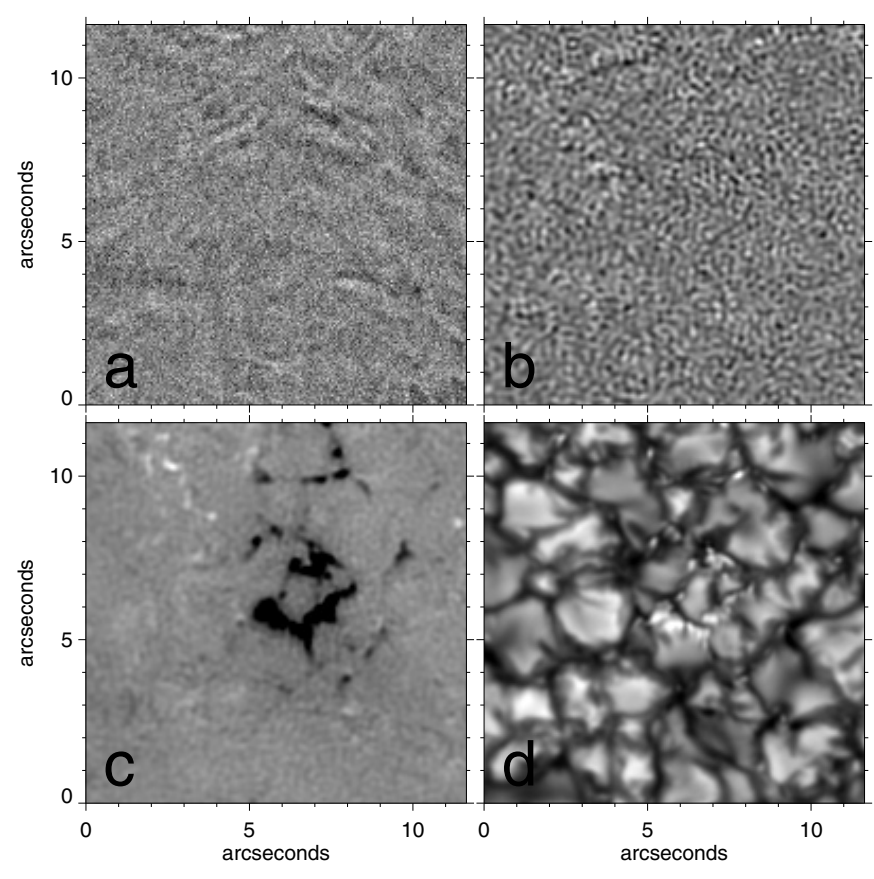

Fig. 6. Panel a) shows a magnetogram-like image constructed from flatfielded images of the same liquid crystal state. Panel b) shows a similar image constructed from a MOMFBD restoration based on the same images as panel a). Both panel a) and b) have the same scaling, -0.01 to +0.01 . Panel c) shows the FeI $630.2-5$ pm Stokes $V$ magnetogram from the same data set and the same number of images. The scaling is -0.05 to +0.05 . Panel d) shows the wide band image of the same region and data set.

Sun that is guaranteed to be completely free of magnetic field. Moreover, observing at a presumably field-free location or at a magnetically insensitive wavelength does not provide polarization-free images due to the instrumental polarization cross-talk. Although the latter can be corrected for using the telescope model, this potentially entangles the polarization crosstalk with the seeing cross-talk, which is undesirable.

Instead, we opted for "re-labeling" the data recorded for one specific modulation state and wavelength to the other states and reducing this dataset as if it were polarized data. Since the signal in all images corresponds to the same state, no polarization signal should be detected after the reductions are complete. By repeating this for all states, a reasonable estimate can be made of the false signal due to for example noise and seeing-crosstalk. Unfortunately, since the polarimeter itself polarizes in Stokes $Q$ and $U$, but not much in $V$, this method could only be used on data that contain only Stokes $V$ observations.

The top panels of Fig. 6 show parts of these "fake" magnetograms constructed using the standard formula $\left(I_{\mathrm{LCP}}-\right.$ $\left.I_{\mathrm{RCP}}\right) /\left(I_{\mathrm{LCP}}+I_{\mathrm{RCP}}\right)$, where $I_{\mathrm{LCP}}$ and $I_{\mathrm{RCP}}$ are from the same state. Panel (a) is constructed from flat-fielded images, panel (b) is based on MOMFBD restored data. Since the FOV shown in these panels is off-center (away from the AO wavefront sensor target area), anisoplanatism can be expected to introduce image shifts and deformations that introduce seeing crosstalk. For the flat-fielded data we observe patterns on granular scales that can be attributed to seeing crosstalk. For the MOMFBD data, these patterns cannot be discerned. This is confirmed by a power spectrum analysis that shows no conspicuous bumps indicating a significant level of false signal due to seeing crosstalk.
The noise pattern in panel (a) and (b) is different: the "orange-peel" pattern for the MOMFBD data has a larger characteristic scale than for the flat-fielded data, which varies on a pixel-to-pixel scale. The characteristic "orange-peel" pattern in the MOMFBD data can be explained by the low-pass filter employed during the restoration: power at the highest frequencies, beyond the diffraction limit and the noise-dominated regime in Fourier space, are attenuated. This leaves a background or noise pattern with a characteristic spatial scale of a few pixels. The noise filter is a fundamental part of the deconvolution conducted in the MOMFBD procedure. It suppresses the noise level slightly below the level expected from photon noise (see below). However, the characteristic noise pattern with a scale close to the diffraction limit poses problems for the detection of small-scale and weak-signal features because it is difficult to unambiguously distinguish noise at that scale from weak signal. In such cases, a time sequence of measurements could validate detection of weak features, provided that they survive sufficiently long.

Uncertainties in the knowledge of the varying photo sensitivity of the detector pixels is another potential source of error. This type of noise is also known as gain table or flat-field noise. The same detector is used for recording all polarization states but the flatfield for each state is found to be different and therefore uncertainty in the flatfield introduces noise. For our observations, flatfields were constructed by adding a large number of exposures, while the telescope is sweeping over the solar disk. Due to the rapid motion and addition of a large number of images, solar features are effectively averaged out. We found that by adding more than 1000 images, the uncertainty in the flatfield decreases to below $10^{-3}$, the level below which this kind of noise becomes negligible.

Figure 7 shows the RMS noise level of several restored data sets as a function of the number of frames per state, for images "restored" using flat-fielding and addition only and for images restored using MOMFBD reduction. The flat-fielded data follows the expected $1 / \sqrt{n}$ behavior well, which confirms that the exposures are dominated by photon noise. The noise level of the MOMFBD restored images is lower than the theoretical $1 / \sqrt{n}$ limit, presumably due to a reduction in high-frequency noise from filtering beyond the diffraction limit.

A number of noise measurements of real Stokes magnetograms are plotted in Fig. 7. These measurements were carried out on small, presumably field-free regions in the FOV. The noise in full Stokes measurements is about two times higher than in observations devised to measure only Stokes $V$. This is because the efficiency in measuring the 4 Stokes parameters is almost four times lower than measuring only Stokes $V$.

For a full Stokes measurement, 125 exposures per state, or 500 exposures in total, is about the maximum number of exposures that can be included if one considers the photospheric evolution time. It takes about $14 \mathrm{~s}$ to acquire these 500 exposures. A disturbance moving at a velocity of the photospheric sound speed $\left(\sim 7 \mathrm{~km} \mathrm{~s}^{-1}\right)$ travels about $100 \mathrm{~km}$ during that time, which is about the distance of the diffraction limit at this wavelength.

Following the same arguments for a measurement of only Stokes $V$, double the amount of exposures per state can be used since the liquid crystals alternate between two states only. In the Stokes $V$ only data set presented in Fig. 7, we measure a noise level of about $2 \times 10^{-3}$ in FeI $630.2-5$ pm. Using the calibration constant of $16551 \mathrm{Mx} \mathrm{cm}^{-2}$ per polarization percent found by Berger et al. (2004) for FeI $630.2-5$ pm SOUP Stokes $V$ magnetograms, this noise level is equivalent to a magnetic flux density of $33 \mathrm{Mx} \mathrm{cm}^{-2}$. 


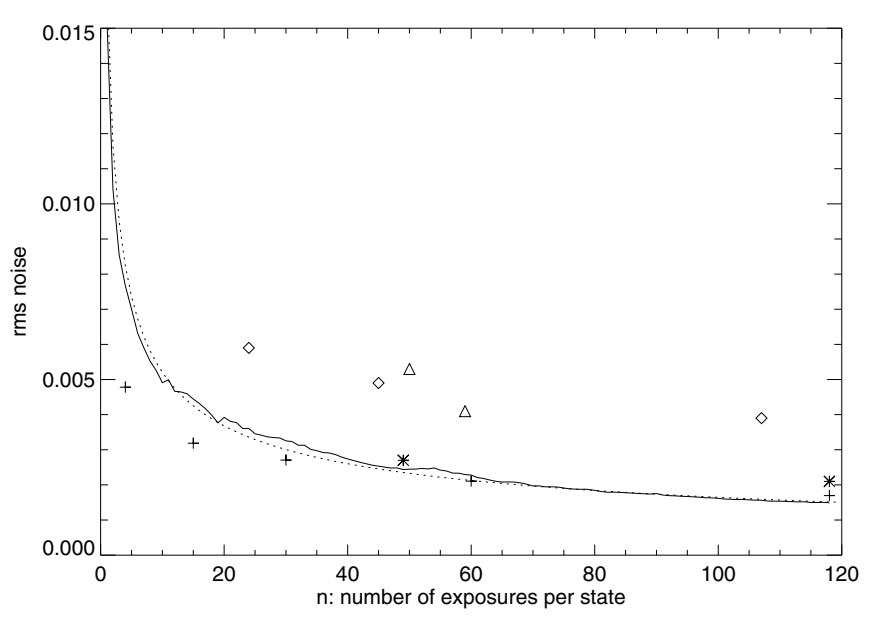

Fig. 7. Noise in "fake" magnetograms as function of number of exposures per state. The solid line is for flatfielded data, similar to panel a) of Fig. 6. Crosses are MOMFBD restored data, similar to panel b) of Fig. 6 . The dotted line is the $1 / \sqrt{n}$ noise behaviour of the photon-noise dominated case with 17 photo-electrons per count. Noise measurements on real $-50 \mathrm{~m} \AA$ magnetograms: quiet Sun Stokes $V$ only (asterisks), Stokes $Q$ close to the sunspot of 12-Sep.-2006 (diamonds), Stokes $U$ disk center from 2007 (triangles).

\subsection{Spatial resolution}

To establish the achieved spatial resolution of the observations, we complete a power spectrum analysis and a close inspection of the smallest resolved structures in the data.

Figure 8 shows sample images of the observations from 12 Sep. 2006. The images shown are based on 110 exposures per state, corresponding to a total of 440 exposures. The data for panel (c) was flat-fielded and added, as if it were obtained using long exposures (1.65 s per state, $6.6 \mathrm{~s}$ effective exposure in total), but with the added advantage that the states were interleaved and therefore the seeing for each of the states was more equal than for equivalent long exposures. The difference in resolution between panel (b) and (c) is striking. The MOMFBD restored Stokes $V$ image shows a lot more fine detail and there are many examples of small, weak features that are not resolved in the flat-fielded stokes $\mathrm{V}$ image.

The power spectra in panel (d) are angular averages of $2 \mathrm{D}$ Fourier power spectra of a $256 \times 256$ pixel sub-area in a corner of the FOV, away from the sunspot and showing only granulation. The power spectrum of the MOMFBD restored wide-band image (based on 440 focus and 440 de-focus images) shows a gradual decrease in power to higher frequencies, with a steeper decline between about $0{ }^{\prime} 18$ and just above the diffraction limit $\lambda / \mathrm{D}$ at $0{ }^{\prime} 134$. The steep decline is an effect of the noise filter that has a smooth transition from no effect at the lower frequencies to full suppression at the highest, noise-dominated, frequencies. The SOUP exposures used to construct the Stokes data are noisier than the wide-band images and therefore the noise filter sets in a lower frequency, corresponding to a spatial scale of about 0.2 . The power spectrum for the flat-fielded Stokes $I$ image is shown for comparison and illustrates how the MOMFBD restoration enhances power at all relevant spatial frequencies. There is a small enhancement in power just above the diffraction limit for the flat-fielded data. That is caused by a small localized power enhancement in the Fourier domain, probably due to some internal camera property. This has no significant effect on the restored data since it is effectively suppressed by the noise filter.

It is difficult to extract a hard number for the achieved spatial resolution from the averaged power spectrum. Even though the steep decline due to the noise filter starts slightly above 0 !' 2 , a claim of a spatial resolution of less than 0.2 in the Stokes data is justified. We performed a close inspection of the smallest features in the Stokes magnetograms and found many examples of structures that had widths with a FWHM of about 3 pixels (0.'19).

\subsection{Unresolved issues}

Even though the most obvious sources of error were discussed above, additional errors from limitations in instrumentation and data processing remain. Although the observing scheme was set to minimize these effects wherever possible, several issues remain unresolved and should be considered when interpreting the data in detail.

\subsubsection{Doppler crosstalk}

Since, for reasons of cadence and detection sensitivity, the data were recorded only at 2 wavelengths, a potentially serious source of false signal is introduced by Doppler shifting of the line profile due to motions in the solar atmosphere. Due to the lack of wavelength information, correcting for this involves assumptions regarding the true line profile.

Although it is possible to remove the polarized part of the line profile using the Stokes inversions, it remains important to remember that the line used here is magnetically sensitive and even the non-polarized profile may have a complicated shape. Even the only plausible assumption of a symmetric line profile may therefore be only of limited value.

Unfortunately, fake magnetogram tests, such as those used in Sect. 4.1, cannot be used to derive an indication of the error made in this way, since the velocity structure evolves on the same time scale as the solar structure, so that the line is affected similarly in both restored images of the set.

A comparative study of the Doppler velocities obtained using a magnetically insensitive spectral line and a magnetically sensitive one, such as FeI 630.2, appears to be the most promising way forward in resolving this issue, but will certainly require more than two wavelength points.

\subsubsection{Restoration effects}

Application of the MOMFBD restoration method enables the removal of a significant amount of seeing-induced aberrations from the observations as well as sub-pixel alignment of the different objects. However, the use of this particular image restoration technique introduces a new kind of variability to the problem caused by the quality of the restoration. This quality is related to the seeing quality and the amount of detail used in the wavefront sensing step of the image restoration process.

In particular, the number of atmospheric wavefront modes (Karhunen-Loève polynomials) used to fit the wavefront is important. The higher order modes do not affect significantly the core structure of the PSF, and therefore the morphology of the restored images, but they have a significant impact on the wings of the PSF, which predominantly affects the contrast of the image. 

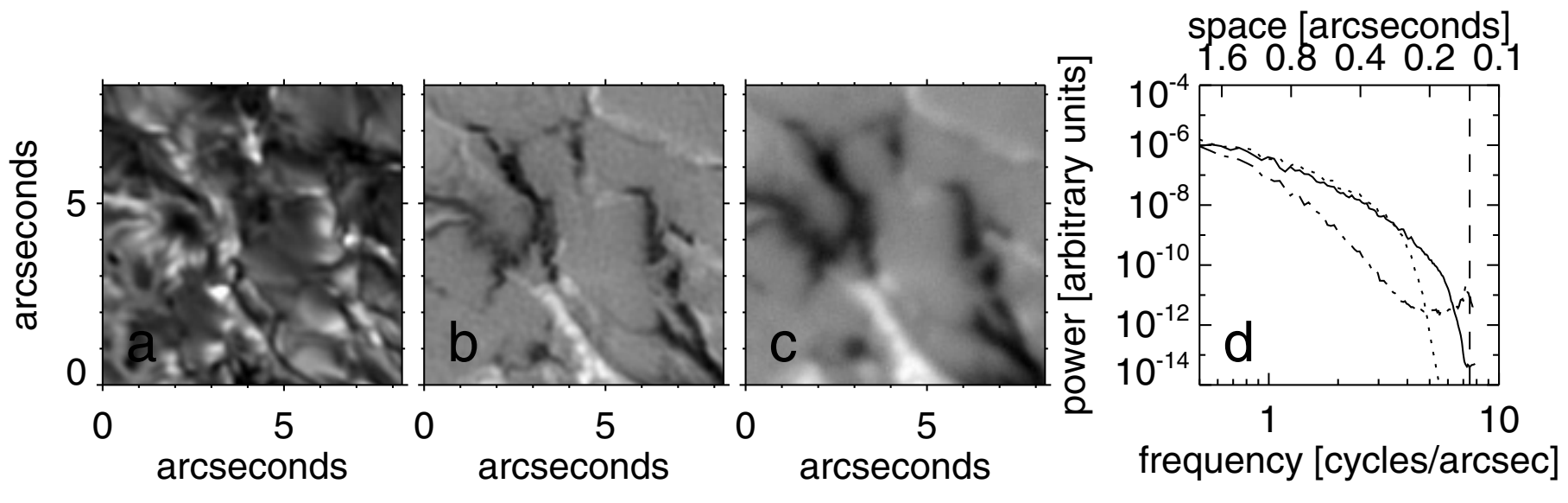

Fig. 8. Panel a) shows a part of a FeI 630.2 -5 pm Stokes $I$ image, observed on 12-Sep-2006, MOMFBD restored based on 110 exposures per state. Panel b) shows the corresponding MOMFBD restored stokes V image. Panel c) is a Stokes $V$ image constructed from the flat-fielded data. Panels b) and c) have the same scaling, -0.2 to 0.2 . The graph in panel d) shows power spectra of part of the MOMFBD restored wide-band image (solid line), the MOMFBD restored Stokes $I$ image (dotted), and the flat-fielded Stokes $I$ image (dash-dotted line). The vertical long-dashed line marks the theoretical diffraction limit $\lambda / \mathrm{D}$ of $0{ }^{\prime} 134$ at this wavelength.
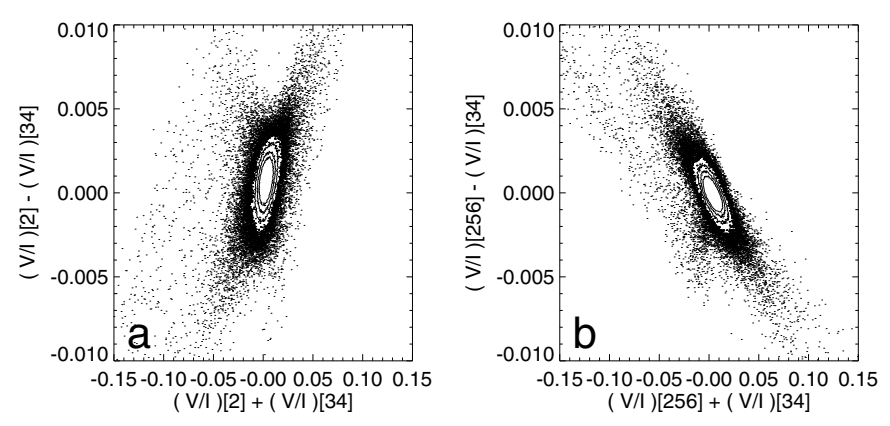

Fig. 9. Scatter plots of relative difference comparing Stokes $V$ magnetograms restored with different number of wavefront modes. Panel a) compares a 2-mode with a 34-mode restoration, b) compares a 256mode with a 34-mode restoration. For the densest scatter regions, density contours are plotted to avoid crowding.

The impact of including higher order wavefront modes on derived quantities, such as Doppler velocity and Stokes maps, is therefore, in general, an increase in the amplitude of the signal. This is illustrated in Fig. 9, which shows plots of the intensity for the same Stokes $V$ magnetogram restored with different numbers of modes.

Panel (a) compares a 2-mode restoration (tip-tilt only) with a 34-mode one, which is the most common number of modes used in MOMFBD restorations. There is a weak correlation, with significant scatter, especially for low-signal pixels. This is consistent with a considerable change in the structure of the image, presumably due to an increase in the resolution of the image caused by the removal of seeing induced aberrations.

Panel (b) shows the same plot but comparing the 34-mode restoration with a 256-mode one. Clearly the correlation for most pixels in the image is tighter and can be fitted to a line with a slope of approximately -0.1 . This is more consistent with a systematic scaling of the 34-mode image to the 256-mode image with a factor of approximately 1.2, than with a substantial change in the image structure.

Although the effect of a scale factor on the fitted values of atmospheric quantities predicted by detailed atmospheric modeling may be quite limited, since the line shape should not be affected, the true error introduced by this effect remains unknown since no modeling was performed on this data.

\section{Results}

\subsection{Dark-cored penumbral filaments}

Dark-cored penumbral filaments (Scharmer et al. 2002) are characterized by bright edges separated by a dark central core. Their symmetrical appearance i.e. limited fine structuring along their length, at times considerable length (over $6000 \mathrm{~km}$, Rouppe van der Voort et al. 2004), and coherent dynamical evolution and lifetime ( $\leq 45 \mathrm{~min}$ reported by Sütterlin et al. (2004) and $\leq 90$ min by Langhans et al. (2007)) suggest that the darkcored filaments are single entities and constitute important building blocks of sunspot penumbrae. An explanation of the existence of dark cores is crucial to the understanding of penumbrae and requires a full observational characterization requiring polarimetric measurements of spatial resolution of about 0.3 or less.

For the sunspots we observed, we clearly resolve the dark cores of penumbral filaments in the Stokes maps. We observe that dark-cored penumbral filaments appear vividly in polarized light, and note that the dark cores exhibit weaker polarization signal than their edges. This is observed readily in the boundary region between the umbra and penumbra, where dark-cored filaments can be unambiguously recognized.

Figure 10 shows examples from several datasets of maps of total circular polarization TCP, total linear polarization TLP, and total polarization TP. Traditionally, these maps are constructed by integration over wavelength. Since we have only 2 wavelength positions, these maps are constructed by summing, $\mathrm{TCP}=\sum\left|V_{i}\right| / I_{i}, \mathrm{TLP}=\sum\left(Q_{i}^{2}+U_{i}^{2}\right)^{1 / 2} / I_{i}$, and $\mathrm{TP}=\sum\left(Q_{i}^{2}+U_{i}^{2}+\right.$ $\left.V_{i}^{2}\right)^{1 / 2} / I_{i}$, where the summation is performed over two wavelengths $i: \lambda_{0} \pm 5 \mathrm{pm}$, where $\lambda_{0}$ is the central wavelength of the average line profile at the observed location on the sun. We observe that the dark cores have weaker signal in all summed maps. This is an indication that dark cores possess weaker magnetic fields than their edges.

The top two rows show maps from a spot close to the center of the solar disk, observed on 12 Sep. 2006. These maps show many clear examples of weaker signal in dark cores. The bottom two rows are from a sunspot observed towards the limb at an angle $\theta=55^{\circ}(\mu=0.58)$. There is an interesting difference between limb-side (bottom row) and center-side penumbra: the center-side TLP map shows only very weak signal whereas the 

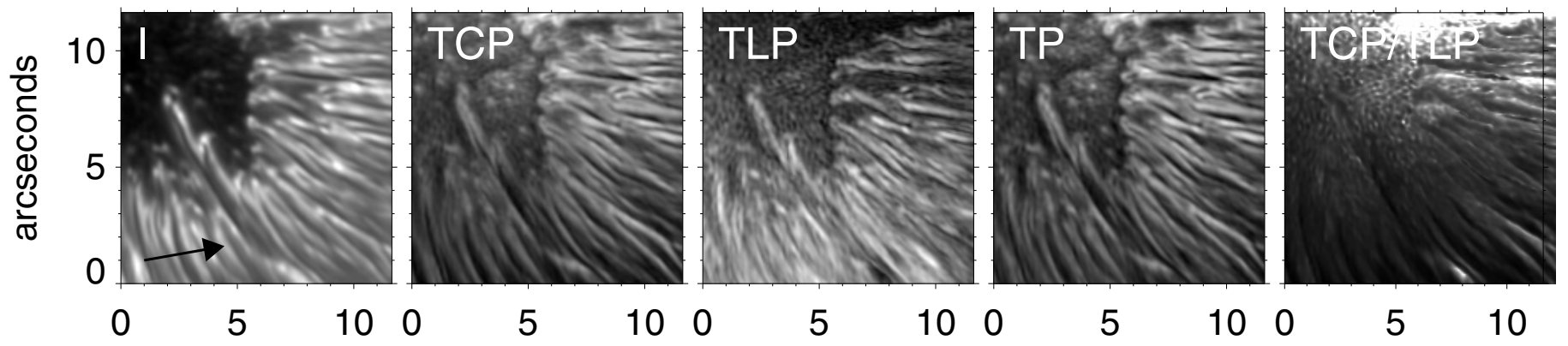

$5 \quad 100$

5

100

5

100

$5 \quad 10$
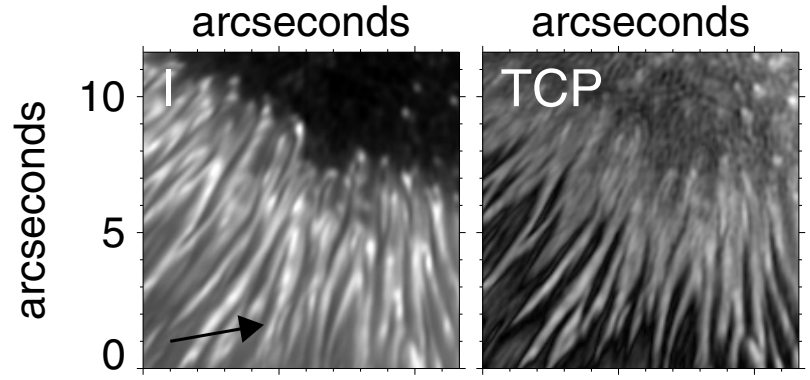

\section{arcseconds}

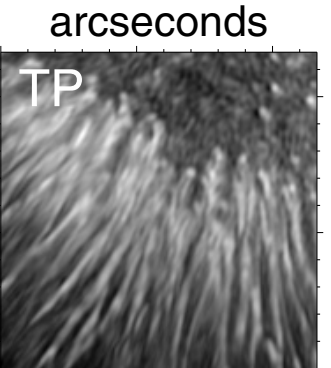

arcseconds

$\begin{array}{llll}0 & 5 & 10 & 0\end{array}$

$5 \quad 100$
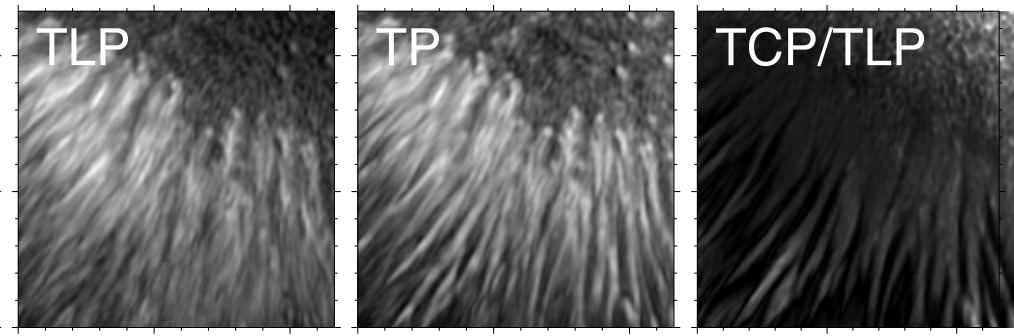

arcseconds

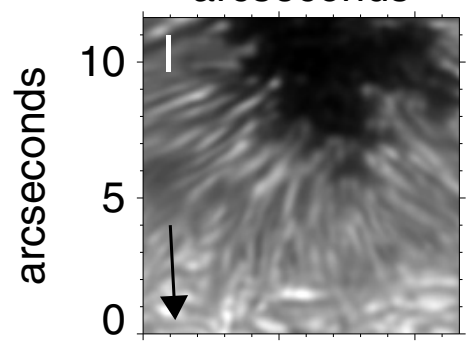

arcseconds

$\begin{array}{lll}5 & 10 & 0\end{array}$

$510 \quad 0$

510

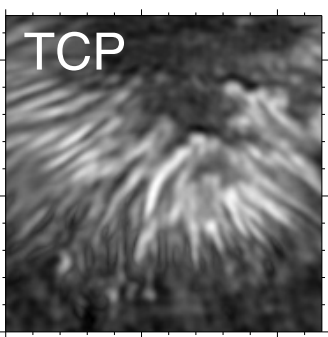

arcseconds

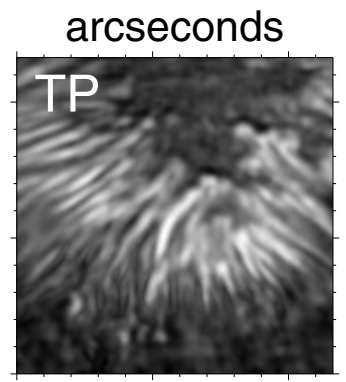

arcseconds
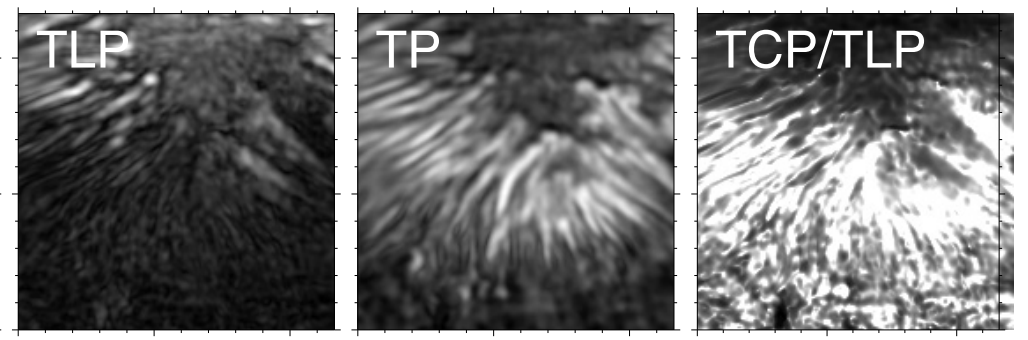

\section{$\begin{array}{cc}0 & 10 \\ \text { arcseconds }\end{array}$}

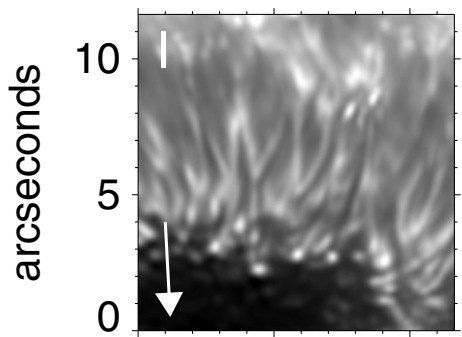

arcseconds

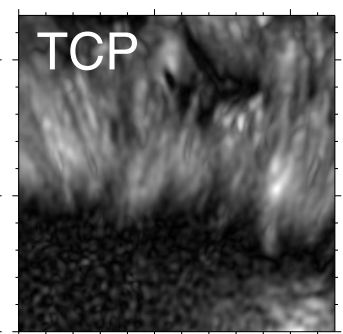

arcseconds

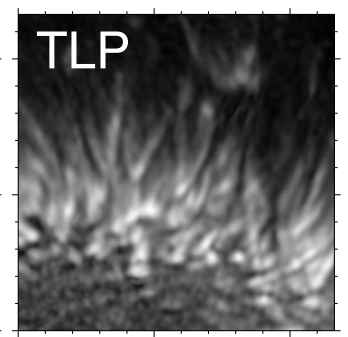

arcseconds

$\begin{array}{lll}0 & 5 & 10\end{array}$
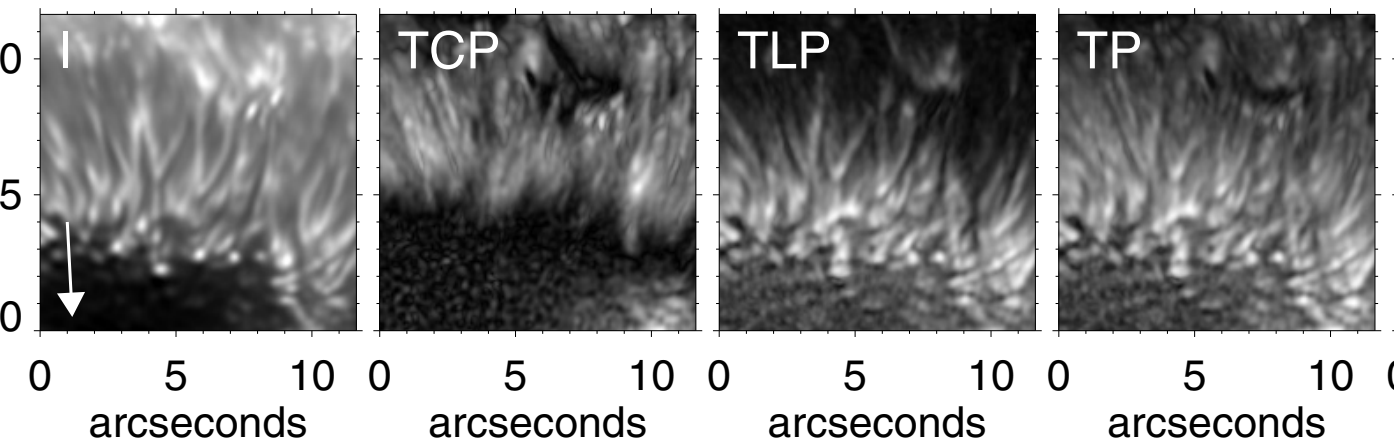

arcseconds

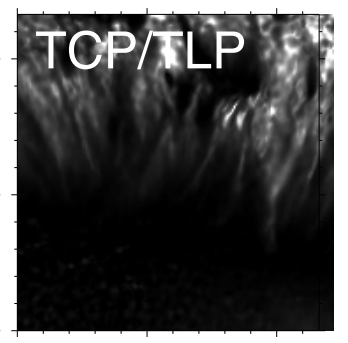

arcseconds

10

arcseconds

Fig. 10. Dark-cored penumbral filaments observed in two sunspots. Top two rows: AR 10908 on 12 Sep. 2006, bottom rows: AR 10908 on 15 Sep. 2006, with the center-side penumbra on the third row, and the limb-side penumbra on the bottom row. Left to right: maps of Stokes $I$ in +5 pm, total circular polarization, total linear polarization, total polarization, and the ratio of total circular over linear polarization. The arrow in the Stokes $I$ images points towards the center of the solar disk.

limb-side TLP map shows distinctively high contrast between dark cores and their edges. This must be interpreted as a line-ofsight (LOS) effect with the magnetic field having small inclination angles with respect to the LOS on the center-side penumbra. This means that the angle of the magnetic field with respect to the solar surface must be about $35^{\circ}$ for the penumbra of this sunspot. That would also explain the relatively weak signal in TCP for the limb-side penumbra compared to the center-side.

In the rightmost column of Fig. 10, maps of the ratio of circular to linear polarization are shown. The dark cores in the top two maps of the sunspot observed on 12 Sep. 2006 appear clearly and they are enhanced in TLP signal compared to their edges (i.e. the dark cores stand out as dark features). This indicates that the magnetic field in dark cores have larger inclination angles with respect to the normal compared to their bright edges. For the center-side TCP/TLP map, the TLP signal is too low to show any significant features. In the limb-side map, the dark cores stand out as bright features. This also is compatible with a more inclined magnetic field in the dark cores with respect to the surface normal, since an increase in inclination in the direction of the penumbral filaments in this part of the penumbra amounts to a decrease in inclination with respect to the LOS and therefore an increase in TCP signal. 

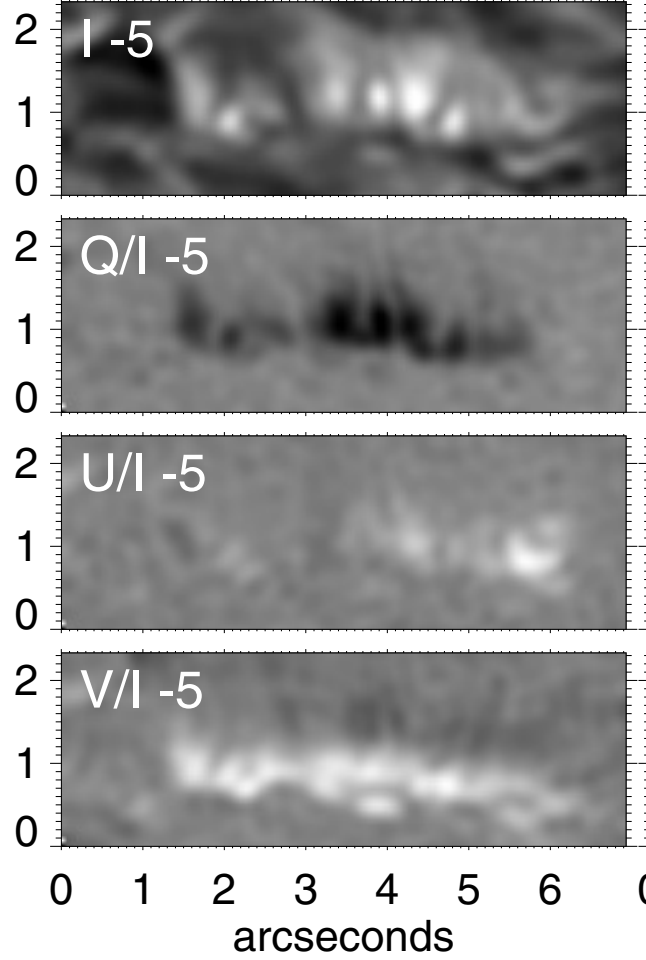
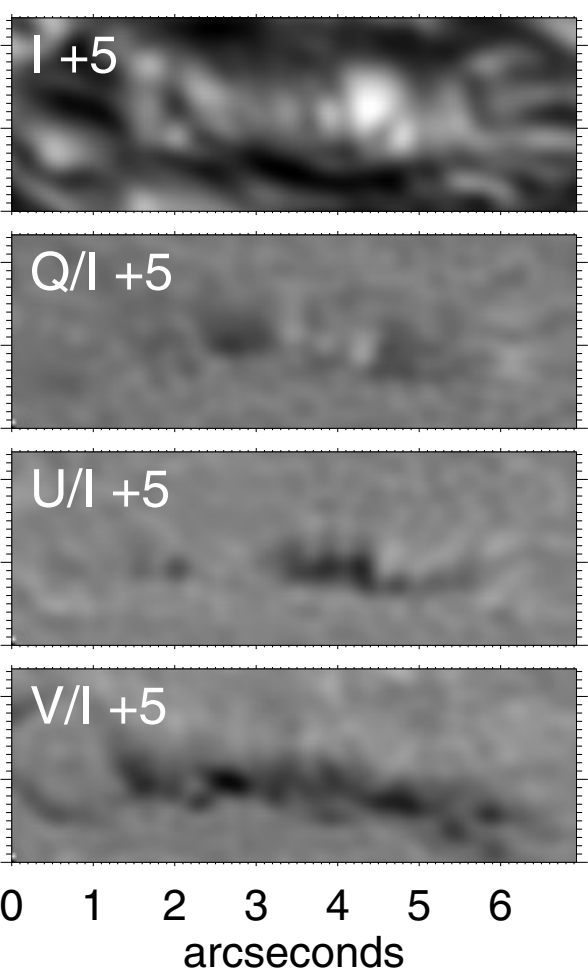
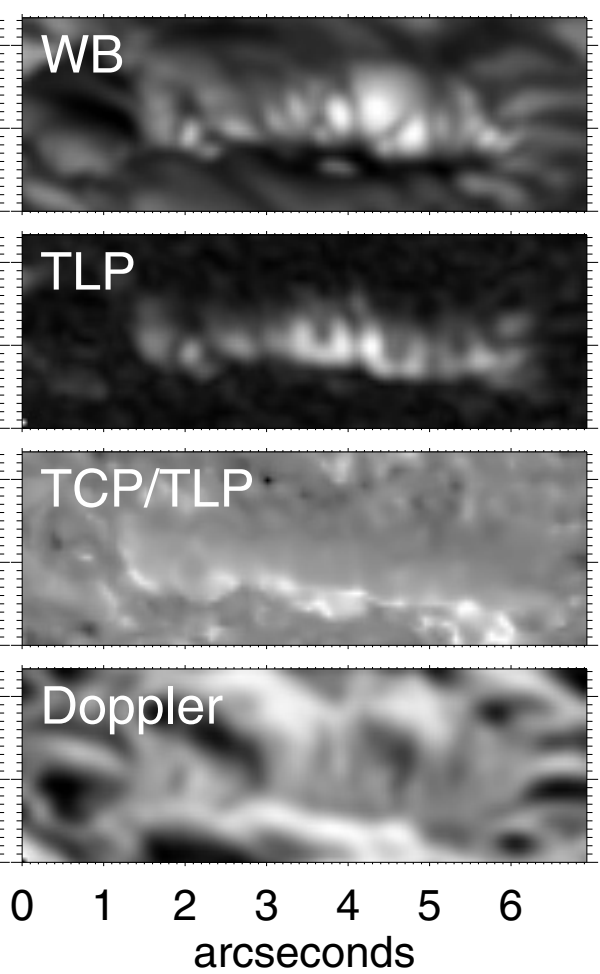

Fig. 11. Faculae observed on 7 Jan. 2006. The left column shows Stokes maps at -5 pm, the middle column at +5 pm. The Stokes maps $Q / I, U / I$, $V / I$ saturate at $\pm 10 \%$. The right column shows from top to bottom: wide-band (WB), total linear polarization (TLP), the ratio of total circular over linear polarization, and a map of the Doppler signal computed from the \pm 5 Stokes I images. The limb direction is towards the top of the figures.

Langhans et al. (2007) concluded on the basis of a geometrical study of high spatial resolution SST Stokes $V$ magnetograms that dark cores possess a weaker magnetic field and that the field is more inclined with respect to the surface normal than their bright edges. We confirm this result using observations that achieve similar resolution, include Stokes $U$ and $Q$ maps and are compensated for Doppler shift effects by using two wavelength positions, one on each side of the central wavelength of the spectral line.

Bellot Rubio et al. (2007) found weaker dark core polarization signals in Hinode spectropolarimetric observations. Their observations had superior wavelength coverage and slightly higher signal-to-noise. Their resolution was about a factor 2 poorer than our observations, which implies that the penumbral dark cores were hardly resolved. Their inversions on the Hinode data indicate that dark cores have weaker and more inclined magnetic fields. From the splitting of the FeII $615 \mathrm{~nm}$ line, Bellot Rubio et al. (2005) deduced that dark cores possess weaker fields than their surroundings.

These observations of weaker signals in dark cores cannot be the basis for a firm conclusion on the magnetic field strength and orientation in dark-cored penumbral filaments. A definite conclusion must be based on thorough modeling that takes the thermodynamic properties of the penumbral atmosphere into account.

\subsection{Faculae}

Another example of the spatial resolution and sensitivity that can be achieved is provided by the facular region surrounding AR10845 observed on 7 Jan. 2006. Since the active region is observed at a large inclination angle $(\mu=0.39)$, the images have low contrast and few structures with spatial scales at or below the diffraction limit. The region shows several clusters of faculae surrounding the main sunspot. Figure 11 shows an overview of the different maps of one such facular cluster.

The wide-band image ("WB") shows the faculae clearly identifiable as a row of bright features, showing striations perpendicular to the limbward direction and a sharp, dark lane on the disk-center side. Such fine-structure in faculae was reported earlier and discussed for high-resolution SST images by Lites et al. (2004) and De Pontieu et al. (2006). The faculae are also clearly observable in the Stokes maps, with a similar degree of fine structuring at the location of the faculae in the circular and linear polarization maps as in Stokes $I$ and wide-band.

In the -5 pm Stokes V/I map, we clearly detect positive signal (white) from magnetic fields pointing towards us at the diskcenter side of the faculae and negative signal (dark) from magnetic fields pointing away from us on the limb side. A similar trend, with opposite sign, is visible in the $+5 \mathrm{pm} \mathrm{V/I} \mathrm{map,} \mathrm{al-}$ though the signals are slightly weaker. This behavior is in agreement with the interpretation of faculae as magnetic flux concentrations, fanning out horizontally over its surroundings when the gas pressure falls below the magnetic pressure.

The TLP map shows the presence of significant field in the plane perpendicular to the LOS in the middle part of the faculae, but it shows almost no signal where the strongest negative signal can be observed in the $-5 \mathrm{pm} V / I$ map. This is probably the region where the magnetic field is pointing only in our direction and is indicative of a well-organized magnetic field structure as described above.

In all of the polarization maps, a clear correlation is observed between the presence of striations and the complete absence of any magnetic signal. In the map of the ratio of circular to linear polarization (TCP/TLP), no variations can be discerned at the location of the striations. This indicates that the decrease in polarization signal is not caused by a variation in the magnetic field 
orientation but by a decrease in magnetic field strength. This is consistent with the explanation of Carlsson et al. (2004) that facular striations are caused by variations in the field strength of the magnetic elements.

The numerical simulations by Keller et al. (2004) and Carlsson et al. (2004) are successful in explaining the detailed morphology of faculae observed at high spatial resolution. The enhanced brightness of faculae are caused by the low density in the atmosphere of magnetic elements compared to the surrounding medium. This allows the observer to look through the magentized atmosphere at the hot wall of the granule directly behind it. The high temperature of the granular wall and the small inclination angle of the LOS with respect to the surface normal of the granule results in a brightening of the parts of granules directly behind magnetic elements compared to their surroundings. Horizontal variations in the magnetic field lead to associated variations in density and opacity, which explains the observation of the striations in the direction perpendicular to the limbward direction. De Pontieu et al. (2006) provided an extended discussion of the spatial and temporal variation of faculae comparing time sequences of high-resolution observations and numerical simulations.

Berger et al. (2007) observed reduced Stokes V/I signal in facular striations using high resolution SST magnetograms. We extend these observations with measurements in linear polarization, which indicate that it is the decrease in the magnetic field that cause striations and not variations in the magnetic field orientation.

The individual $Q / I$ and $U / I$ maps, show significantly different behavior on the red and the blue side of the line center, although the line profile is naively expected to be symmetric. Notably, in the red wing of the line the polarity of the Q/I and $\mathrm{U} / \mathrm{I}$ signals is seen to change sign repeatedly in the same row of faculae, whereas it does not change sign in the blue wing.

The 2-point Dopplermap shows that there is some variation in the Doppler signal across the faculae. This indicates that the asymmetry between the red and blue wing maps might be caused by velocities along the line of sight. Without additional spectral information available, the cause of this asymmetry, or why it is particularly irregular in the red side of the line, remains unclear.

\section{Discussion}

We have obtained near diffraction-limited full Stokes images of the solar surface, with a $\mathrm{S} / \mathrm{N}$ of $2 \times 10^{-3}$ in Stokes $V$ only mode and $4 \times 10^{-3}$ in full Stokes mode, corresponding to a noise level of $\approx 1.3 \times 10^{-3}$ and $\approx 2.5 \times 10^{-3}$ compared to the continuum intensity, a more conventional quantity used in spectropolarimetry. This translates into a maximum sensitivity $33 \mathrm{Mx} \mathrm{cm}-2$ and $66 \mathrm{Mx} \mathrm{cm}^{-2}$, respectively, in the weak field approximation. Although only 2 wavelengths were observed, this data demonstrates that the acquisition of diffraction-limited, high $\mathrm{S} / \mathrm{N}$ fullStokes images using $1 \mathrm{~m}$-class ground-based solar telescopes is achievable.

The observed features in penumbral dark cores and faculae are consistent with the results of numerical modeling and evidence from Stokes $V$ only data.

Despite the high quality of the results, we note that there are at least two major limitations in our current observing system that could be improved significantly in terms of sensitivity and accuracy.

The SOUP filter is a Lyot filter, which means that it has a linear polarizer that transmits only half of the incoming light. Due to the large number of optical elements and the way in which it filters the light, actual filter transmission is, at $\sim 15 \%$, well below that of any good quality modern filter, which may have 4-5 times higher transmission. With a pre-filter transmission of $\approx 50 \%$, the total transmission is only $\approx 7 \%$. Moreover, the SOUP filter has a relatively long line position change time of $\approx 6 \mathrm{~s}$, implying that in our current scheme of $\approx 14 \mathrm{~s}$ acquisition time per line position, $40 \%$ of the available photon collection time is lost by filter tuning. A modern piezo-tuned filter system can typically tune in a matter of a few ms, so that it can change line position during the readout time of the camera. Since this completely eliminates the time lost by filter tuning, an improved acquisition scheme, with the filter states and the LCVR states interleaved, then becomes possible, with all the associated benefits in terms of homogenizing the seeing conditions for all states. Furthermore, by cycling continuously through a number of line positions, one can decide a-posteriori what time scale is acceptable for the features to be studied and apply image restoration on a dataset covering the appropriate fraction of that feature's evolution time.

The readout time of the Sarnoff cameras is fixed, at $10 \mathrm{~ms}$, so that with an exposure time of $15 \mathrm{~ms}$ and a compulsory "dead" time of $2 \mathrm{~ms}$ related to the chip size and the shutter speed, a total of $45 \%$ of the available photon collection time is lost. High-speed frame-transfer cameras would enable an additional increase in efficiency of approximately a factor 2 , but reduce the possibility of interleaving filter states, due to the finite tuning times of LCVRs and narrow-band filters.

The overall efficiency of the current setup is estimated to be a mere $2-3 \%$, a figure that can surely be improved upon.

Apart from the efficiency aspect, the filter tuning time limits the possible number of line positions. With a tuning time of several seconds, the evolution time of the Sun does not permit more than a few wavelength points. An increased number of line positions would, however, provide a highly desired enhanced polarimetric accuracy compared with the current two, as discussed in detail by Graham et al. (2002).

A Fabry-Perot Interferometer (see e.g. IBIS (Cavallini et al. 2003; Reardon \& Cavallini 2003), and TESOS (Kentischer et al. 1998)) is a system that can decrease some of the shortcomings of the current setup. Such an instrument was installed at the SST (see Scharmer (2006) for a design study), the transmission of which is 3-4 times higher than for the SOUP filter and in addition a polarizing beamsplitter is used to ensure maximum use of the available light. Moreover, changing line position can be accomplished in less than a camera readout time $(<10 \mathrm{~ms})$, allowing multiple line positions to be covered in a fraction of a second. The overall increase in performance is therefore expected to yield significantly more accurate results than possible with the instruments used here.

Acknowledgements. The Swedish 1-m Solar Telescope is operated on the island of La Palma by the Institute for Solar Physics of the Royal Swedish Academy of Sciences in the Spanish Observatorio del Roque de los Muchachos of the Instituto de Astrofísica de Canarias. This research was supported through grants $146467 / 420$ and $159137 /$ V30 of The Research Council of Norway. We thank Bruce Lites for his invaluable contributions to the development of the polarimetry calibration procedure of the SST. This research has made use of NASA's Astrophysics Data System.

\section{References}

Beck, C., Schlichenmaier, R., Collados, M., Bellot Rubio, L., \& Kentischer, T. 2005a, A\&A, 443, 1047

Beck, C., Schmidt, W., Kentischer, T., \& Elmore, D. 2005b, A\&A, 437, 1159 Bello González, N., \& Kneer, F. 2008, A\&A, 480, 265

Bellot Rubio, L. R., Langhans, K., \& Schlichenmaier, R. 2005, A\&A, 443, L7 Bellot Rubio, L. R., Tsuneta, S., Ichimoto, K., et al. 2007, ApJ, 668, L91 
Berger, T. E., Rouppe van der Voort, L. H. M., Löfdahl, M. G., et al. 2004, A\&A, 428,613

Berger, T. E., Rouppe van der Voort, L., \& Löfdahl, M. 2007, ApJ, 661, 1272

Carlsson, M., Stein, R. F., Nordlund, Å., \& Scharmer, G. B. 2004, ApJ, 610, L137

Cavallini, F., Baffa, C., Reardon, K., et al. 2003, Mem. Soc. Astron. It., 74, 796

Collados, M., Lagg, A., Díaz Garcí A, J. J., et al. 2007, in The Physics of Chromospheric Plasmas, ed. P. Heinzel, I. Dorotovič, \& R. J. Rutten, ASP Conf. Ser., 368, 611

De Pontieu, B., Carlsson, M., Stein, R., et al. 2006, ApJ, 646, 1405

De Pontieu, B., Hansteen, V. H., Rouppe van der Voort, L., van Noort, M., \& Carlsson, M. 2007, ApJ, 655

Gandorfer, A. M., \& Povel, H. P. 1997, A\&A, 328, 381

Graham, J. D., López Ariste, A., Socas-Navarro, H., \& Tomczyk, S. 2002, Sol. Phys., 208, 211

Hale, G. E. 1908, ApJ, 28, 315

Horn, T., \& Hofmann, A. 1999, in Third Advances in Solar Physics Euroconference: Magnetic Fields and Oscillations, ed. B. Schmieder, A. Hofmann, \& J. Staude, ASP Conf. Ser., 184, 33

Keller, C. U., \& von der Lühe, O. 1992, A\&A, 261, 321

Keller, C. U., Schüssler, M., Vögler, A., \& Zakharov, V. 2004, ApJ, 607, L59

Kentischer, T. J., Schmidt, W., Sigwarth, M., \& Uexkuell, M. V. 1998, A\&A, 340,569

Langhans, K., Scharmer, G. B., Kiselman, D., \& Löfdahl, M. G. 2007, A\&A, 464, 763

Lites, B. W., Elmore, D. F., \& Streander, K. V. 2001, in Advanced Solar Polarimetry - Theory, Observation, and Instrumentation, ed. M. Sigwarth, ASP Conf. Ser., 236, 33

Lites, B. W., Scharmer, G. B., Berger, T. E., \& Title, A. M. 2004, Sol. Phys., 221, 65

Mártinez Pillet, V., Collados, M., Sánchez Almeida, J., et al. 1999, in High Resolution Solar Physics: Theory, Observations, and Techniques, ed. T. R. Rimmele, K. S. Balasubramaniam, \& R. R. Radick, ASP Conf. Ser., 183, 264
Paletou, F., \& Molodij, G. 2001, in Astronomical Society of the Pacific Conference Series, Vol. 236, Advanced Solar Polarimetry - Theory, Observation, and Instrumentation, ed. M. Sigwarth, ASP Conf. Ser., 236, 9

Reardon, K., \& Cavallini, F. 2003, Mem. Soc. Astron. It., 74, 815

Rouppe van der Voort, L. H. M., Löfdahl, M. G., Kiselman, D., \& Scharmer, G. B. 2004, A\&A, 414, 717

Sanchez Almeida, J., Collados, M., Martinez Pillet, V., et al. 1997, in 1st Advances in Solar Physics Euroconference. Advances in Physics of Sunspots, ed. B. Schmieder, J. C. del Toro Iniesta, \& M. Vazquez, ASP Conf. Ser., 118, 366

Sankarasubramanian, K., Gullixson, C., Hegwer, S., et al. 2004, in Presented at the Society of Photo-Optical Instrumentation Engineers (SPIE) Conference, Vol. 5171, Telescopes and Instrumentation for Solar Astrophysics. ed. S. Fineschi, \& M. Gummin, A. Proc. SPIE, 5171, 207

Scharmer, G. B. 2006, A\&A, 447, 1111

Scharmer, G. B., Gudiksen, B. V., Kiselman, D., Löfdahl, M. G., \& Rouppe van der Voort, L. H. M. 2002, Nature, 420, 151

Scharmer, G. B., Bjelksjö, K., Korhonen, T. K., Lindberg, B., \& Petterson, B. 2003a, in Innovative Telescopes and Instrumentation for Solar Astrophysics., ed. S.L. Keil \& S.V. Avakyan, Proc. SPIE., 4853, 341

Scharmer, G. B., Dettori, P. M., Löfdahl, M. G., \& Shand, M. 2003b, in Innovative Telescopes and Instrumentation for Solar Astrophysics. ed. S. L. Keil, \& S. V. Avakyan, Proc. SPIE., 4853, 370

Selbing, J. 2005, Stockholm University Masters Thesis, 613

Skumanich, A., Lites, B. W., Martinez Pillet, V., \& Seagraves, P. 1997, ApJS, 110,357

Suematsu, Y., Tsuneta, S., Ichimoto, K., et al. 2008, Sol. Phys., 26

Sütterlin, P., Bellot Rubio, L. R., \& Schlichenmaier, R. 2004, A\&A, 424, 1049

Title, \& Rosenberg. 1981, Optical Engineering, 20, 815

van Noort, M., Rouppe van der Voort, L., \& Löfdahl, M. G. 2005, Sol. Phys., 228,191 